\title{
Mass Imbalance Compensation Control for Stabilized Platform Based on UKF Identification
}

\author{
Zhang WeiMing ${ }^{1,2,3,4}$, SHI ZeLin ${ }^{1,3,4}$ \\ ${ }^{1}$ Shenyang Institute of Automation, Chinese Academy of Sciences, Shenyang, 110016, China \\ ${ }^{2}$ University of Chinese Academy of Sciences, Beijing. 100049, China \\ ${ }^{3}$ Key Laboratory of Opto-Electronic Information Processing CAS, Shenyang, 110016, China \\ ${ }^{4}$ The Key Lab of Image Understanding and Computer Vision, Liaoning Province, Shenyang, 110016, China
}

\begin{abstract}
Due to the mass imbalance about the center of rotation, the stability of stabilized platform system degrades with carrier's disturbances. Various feed-forward control methods are provided by reaserchers to solve this problem, however these methods are not well applied because the eccentricity of stabilized platform could not be measured directly. The dynamics model of a typical 2-axis stabilized platform is given. The eccentricity vector is identified through Unscented Kalman Filter(UKF) algorithm. Imbalance torque is precisely observed so that the real-time nonlinear compensation for mass imbalance is achieved through a feed-forward loop. The simulation result indicates that the Root Mean Squared Error (RMSE) of parameters estimation is 0.024 after convergence. the LOS stabilization with carrier's $2.5 \mathrm{~Hz}$ vibration is $0.04 \mathrm{rad} / \mathrm{s}$, which improves $78 \%$ compared to conventional feed-back control.
\end{abstract}

\section{Introduction}

There are several effects inherent to the stabilized platform that could have a marked influence on overall pointing and tracking performance, such as noise, random error, and disturbance torque. Among these factors, the most complex nonlinear phenomenon is the effective torque due to mass imbalance and gimbal friction[1], [2], [3], [6].

When the center of gravity (CG) of the gimbal frame deviates from the rotation center, the imbalance torque is generated due to the acceleration in the direction perpendicular to the eccentricity. The line-of-sight (LOS) stabilization degrades seriously when the vibration of carrier is reinforced by high speed airflow or other environment factors.

A conventional approach is to increase the stabilizing loop gain or insert proportional-integral cascaded compensator[7]. However, these methods may not work well for dynamic, highly nonlinear disturbances, or even increase loop sensitivity to sensor noise. If the imbalance torque is observed, feed-forward controller could be introduced to compensate the imbalance effects[8], [9].

\section{Dynamics model of stabilized platform}

Two processes should be considered in the dynamics model of the stabilized platform system, the first is the disturbance transmitting process from the carrier to the payload, the second is the controlling process. A typical 2-axis stabilized platform is given (see fig.1). $O X_{B} Y_{B} Z_{B}$ is the carrier coordinate system in which $X_{B}$ is the spin axis, $Y_{B}$ is the yaw axis and $Z_{B}$ is the pitch axis. $O X_{O} Y_{O} Z_{O}$ is defined when the carrier coordinate system rotate the angle $\psi$ with $Z_{B} . O X_{I} Y_{I} Z_{I}$ is defined when $O X_{O} Y_{O} Z_{O}$ rotate the angle $\theta$ with $Y_{O}$. The origin of these coordinate system coincides with the intersection of the gimbal axis system.

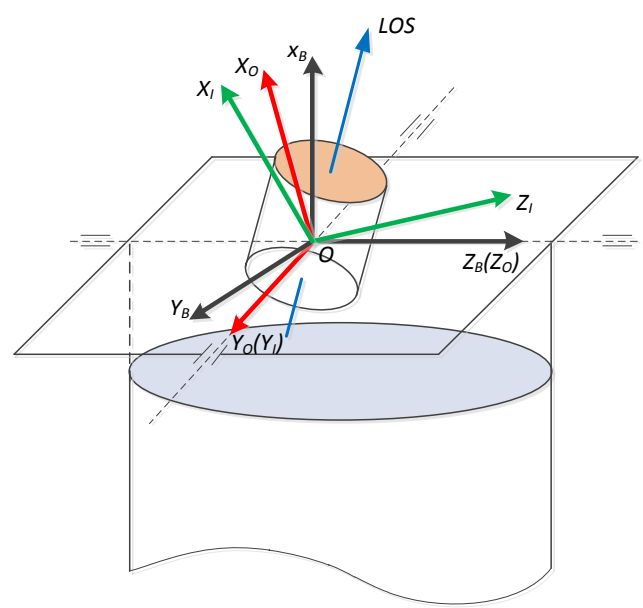

Figure 1. 2-axis stabilized platform.

According to kinematics relationship the inertial LOS rate is given by: 


$$
\boldsymbol{\omega}_{\boldsymbol{I}}=\left[\begin{array}{c}
\omega_{O x} \cos \theta-\omega_{O z} \sin \theta \\
\omega_{O y}+\dot{\theta} \\
\omega_{O x} \sin \theta+\omega_{O z} \cos \theta
\end{array}\right]
$$

The torque of the gimbal axis is:

$$
\boldsymbol{M}_{r}=\frac{\mathrm{d} \boldsymbol{H}}{\mathrm{d} t}+\boldsymbol{\Omega} \times \boldsymbol{H}+\boldsymbol{\rho} \times m \boldsymbol{a}
$$

In equation (2), $\boldsymbol{H}=\boldsymbol{J} \boldsymbol{\omega}$ is the angular momentum of the frame, $\boldsymbol{\rho}$ is the eccentricity vector, $m$ is the frame mass, and $\boldsymbol{a}$ is the acceleration of the frame. The torque $\boldsymbol{M}_{r}$ contains the control torque and other torque disturbances such as coulomb and viscous friction and cable damping effects.

Take the inner frame's situation as example, according to kinematics relationship the coupled acceleration from the carrier is given by:

$$
\left[\begin{array}{l}
a_{I x} \\
a_{I y} \\
a_{I z}
\end{array}\right]=\left[\begin{array}{c}
a_{B x} \cos \psi \cos \theta+a_{B y} \sin \psi \cos \theta-a_{B z} \sin \theta \\
-a_{B x} \sin \psi+a_{B y} \cos \psi \\
a_{B x} \cos \psi \sin \theta+a_{B y} \sin \psi \sin \theta+a_{B z} \cos \theta
\end{array}\right]
$$

The imbalance torque of inner frame is given by:

$$
\begin{aligned}
M_{i m b}= & \rho_{I z} m_{I}\left(a_{B x} \cos \psi \cos \theta+a_{B y} \sin \psi \cos \theta-a_{B z} \sin \theta\right) \\
& -\rho_{I x} m_{I}\left(a_{B x} \cos \psi \sin \theta+a_{B y} \sin \psi \sin \theta+a_{B z} \cos \theta\right)
\end{aligned}
$$

\section{Feed-forward compensation design}

If the imbalance torque is observed, feed-forward compensation could be possible to achieve (see fig.2), and the feed-forward controller is designed as: $G_{N}=-M_{I_{-} i m b} / G_{c l} C_{m}$.It is hard to precisely measure the eccentricity vector of the platform system, for the reason that the most important part of feed-forward compensation designing is the eccentricity identification.

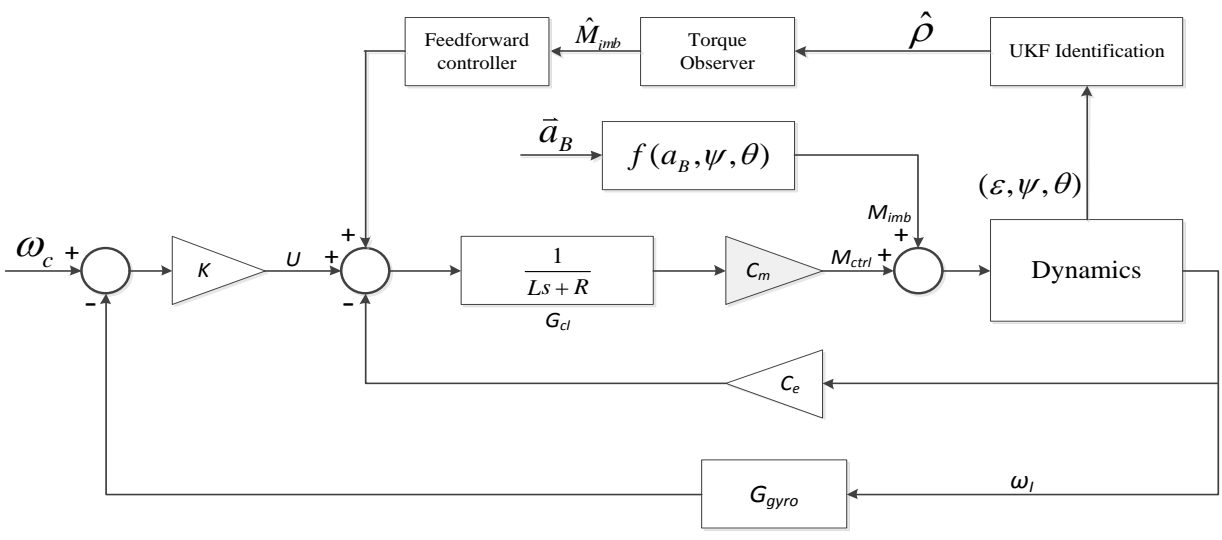

Figure 2. Principle of feed-forward compensation.

Because the relationship between the states and the observations of platform system is nonlinear, it is suitable for using UKF algorithm to estimate the eccentricity [4], [5]. The relationship between the angle acceleration and the eccentricity is:

$$
\begin{aligned}
\varepsilon_{I} & =\rho_{I x} m_{I}\left(a_{B x} \cos \psi \sin \theta+a_{B y} \sin \psi \sin \theta+a_{B z} \cos \theta\right) / J_{I} \\
& -\rho_{I z} m_{I}\left(a_{B x} \cos \psi \cos \theta+a_{B y} \sin \psi \cos \theta-a_{B z} \sin \theta\right) / J_{I}
\end{aligned}
$$

The state vector is given by:

$$
\boldsymbol{X}=\left[\begin{array}{lllllll}
\rho_{I x} & \rho_{I z} & a_{B x} & a_{B y} & a_{B z} & \psi & \theta
\end{array}\right]^{\mathrm{T}}
$$

The measurement vector is given by:

$$
\boldsymbol{Z}=\left[\begin{array}{llllll}
\varepsilon_{I} & a_{B x} & a_{B y} & a_{B z} & \psi & \theta
\end{array}\right]^{\mathrm{T}}
$$

The initialization of UKF is:

$$
\left\{\begin{array}{l}
\hat{X}_{0}=\mathrm{E}\left[X_{0}\right] \\
P_{0}=\mathrm{E}\left[\left(X_{0}-\hat{X}_{0}\right)\left(X_{0}-\hat{X}_{0}\right)^{\mathrm{T}}\right]
\end{array}\right.
$$

And the sigma points are calculated as:

$$
\chi_{k-1}^{a}=\left[\begin{array}{lll}
\hat{X}_{k-1}^{a} & \hat{X}_{k-1}^{a}+\gamma \sqrt{P_{k-1}^{a}} & \hat{X}_{k-1}^{a}-\gamma \sqrt{P_{k-1}^{a}}
\end{array}\right]
$$

The time-update equations are:

$$
\left\{\begin{array}{l}
\chi_{k \mid k-1}^{x}=\mathbf{F}\left(\chi_{k-1}^{x}, u_{k-1}, \chi_{k-1}^{v}\right) \\
\hat{X}_{k}^{-}=\sum_{i=0}^{2 L} W_{i}^{(m)} \chi_{i, k \mid k-1}^{x} \\
P_{k}^{-}=\sum_{i=0}^{2 L} W_{i}^{(c)}\left(\chi_{i, k \mid k-1}^{x}-\hat{X}_{k}^{-}\right)\left(\chi_{i, k \mid k-1}^{x}-\hat{X}_{k}^{-}\right)^{\mathrm{T}} \\
Z_{k \mid k-1}=\mathbf{H}\left(\chi_{k \mid k-1}^{x}, \chi_{k-1}^{n}\right) \\
\hat{Z}_{k}^{-}=\sum_{i=0}^{2 L} W_{i}^{(m)} Z_{i, k \mid k-1}
\end{array}\right.
$$


And the measurement-update equations are:

$$
\left\{\begin{array}{l}
P_{\bar{Z}_{k} \bar{Z}_{k}}=\sum_{i=0}^{2 L} W_{i}^{(c)}\left(Z_{i, k \mid k-1}-\hat{X}_{k}^{-}\right)\left(Z_{i, k \mid k-1}-\hat{Z}_{k}^{-}\right)^{\mathrm{T}} \\
P_{X_{k} Z_{k}}=\sum_{i=0}^{2 L} W_{i}^{(c)}\left(\chi_{i, k \mid k-1}-\hat{Z}_{k}^{-}\right)\left(Z_{i, k \mid k-1}-\hat{Z}_{k}^{-}\right)^{\mathrm{T}} \\
K_{k}=P_{X_{k} Z_{k}} P_{\bar{Z}_{k} \bar{Z}_{k}}^{-1} \\
\hat{X}_{k}=\hat{X}_{k}^{-}+K_{k}\left(Z_{k}-\hat{Z}_{k}^{-}\right) \\
P_{k}=P_{k}^{-}-K_{k} P_{\bar{Z}_{k} \bar{Z}_{k}} K_{k}^{T}
\end{array}\right.
$$

where $X^{a}=\left[\begin{array}{lll}X^{\mathrm{T}} & v^{\mathrm{T}} & n^{\mathrm{T}}\end{array}\right]^{\mathrm{T}}, \chi^{a}=\left[\begin{array}{lll}\left(\chi^{x}\right)^{\mathrm{T}} & \left(\chi^{v}\right)^{\mathrm{T}} & \left(\chi^{n}\right)^{\mathrm{T}}\end{array}\right]^{\mathrm{T}}$, $\gamma=\sqrt{L+\lambda}$.

The eccentricity is extracted from state estimation vector after every filter step, and is used to calculate the feed-forward control command.

\section{Simulation analysis}

According to the system dynamics the simulation model is founded. And the control parameters are given: the motor inductance is $2.8 \mathrm{mH}$, the coil resistance is $5.1 \Omega$, the torque coefficient is 0.21 , the feed-back control gain is 34.5 , the filter step is $0.01 \mathrm{~s}$, and the vibration of the carrier forms a sinusoidal signal with amplitude of $0.5 \mathrm{~g}$ and frequency of $2.5 \mathrm{~Hz}$.

The simulation result indicates that the convergence time of UKF algorithm is $<0.4 \mathrm{~s}$, and the Root Mean Squared Error (RMSE) of parameters estimation is 0.024 after convergence(see Fig.3-4). the LOS stabilized accuracy with carrier's vibration is $0.04 \mathrm{rad} / \mathrm{s}$, which improves 78\% compared to feedback control (see Fig.5-6).

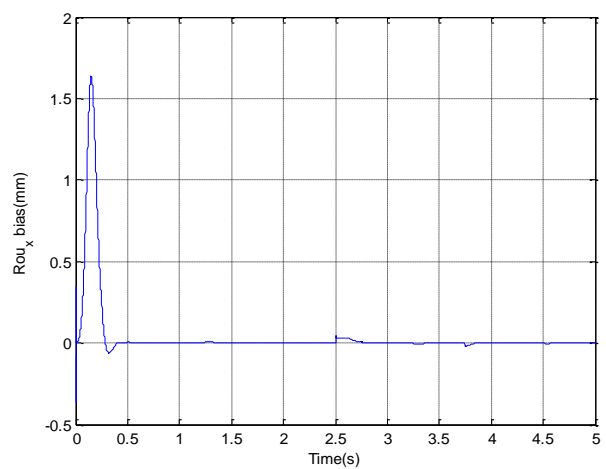

Figure 3. Estimation error of eccentricity in $x$.

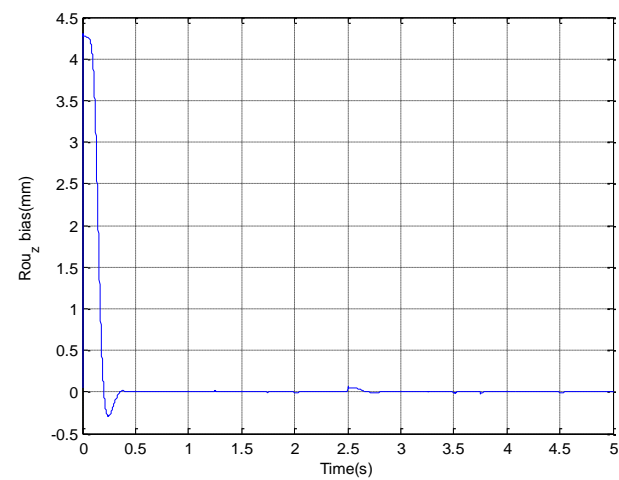

Figure 4. Estimation error of eccentricity in $\mathrm{z}$.

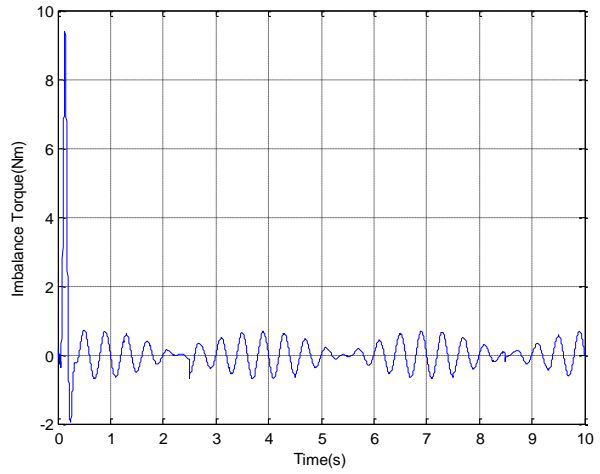

Figure 5. Observed imbalance torque.

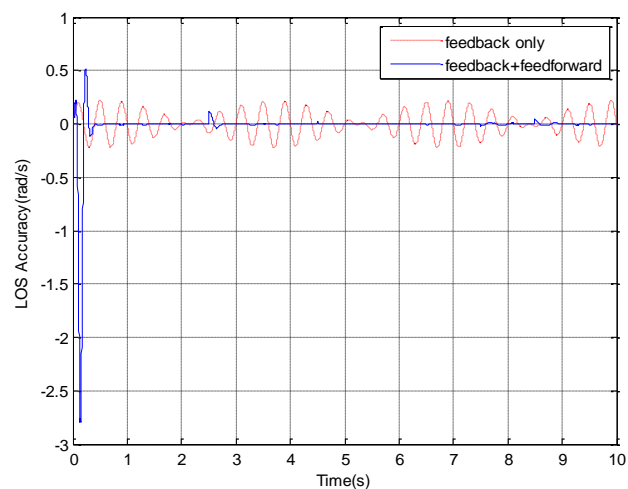

Figure 6. LOS stability comparison.

\section{Summary}

Because measuring a stabilized platform's eccentricity by conventional instruments is almost impossible, an realtime method of identification is provided. The eccentricity vector is precisely estimated through UKF algorithm, so that the imbalance torque could be completely observed. The convergence time of UKF is very short, and the algorithm consumes few hardware resources. The feed-forward compensation is designed which is effective to suppress the disturbances from the carrier, and better LOS stabilization of platform system is achieved compared to the traditional simple feed-back control method.

\section{References}

1. $\mathrm{C}$ L Lin, $\mathrm{Y} \mathrm{H}$ Hsiao, Adaptive feedforward control for disturbance torque rejection in seeker stabilizing loop. IEEE Transaction on Control Systems Technology, 2001. 9(1):108-111.

2. Gong W, Z Cai, J Yang, X Li, L Jian. Parameter identification of an SOFC model with an efficient adaptive differential evolution algorithm. International Journal of Hydrogen Energy, 2014. 39(10): 5083-5096

3. Lu W, L Liu, J Sun, Y Zhou, Y Wu. Analysis of complex axis control loop in satellite laser communications. Optik 2012. 123: 458-461

4. YU H, WANG $\mathrm{G} \mathrm{H}$, SUN $\mathrm{Y}$, Particle filtering algorithm of state estimation on fusion of UKF and 
EKF. Systems Engineering and Electronics. 1001506X(2003)07-1375-05.

5. Andreas S, Hans A. Bridging the ensemble Kalman filter and particle filters: the adaptive Gaussian mixture filter. Computational Geosciences, 2011, 15(2): 293-305.

6. Zhang Y P, Wang W X. Analysis on the stability of visual axis of airborne high precision stabilized platform. Foreign electronic measurement technology, 2015, 34(11):40-44.
7. Peter J Kennedy, Rhonda L Kennedy. Direct versus indirect line of sight (LOS) stabilization[J]. IEEE Transactions on Control Systems Technology, Vol. 11, NO. 1, January 2003:1063-1066.

8. Wei W, Dai M, Li J Q. ADRC control system for airborne opto-electronic platform. Optics and Precision Engineering. 2015,23 (8) :2296-2299.

9. Han Z J. Adaptive control. Beijing: Tsinghua University Press, 2002: 151-158. 